\title{
On the origin and removal of interference patterns in coated multimode fibres
}

Padilla Michel, Yazmin, Pulwer, Silvio, Saffari, Pouneh, Ksianzou, Viachaslau, Schrader, Sigurd

Yazmin Padilla Michel, Silvio Pulwer, Pouneh Saffari, Viachaslau Ksianzou, Sigurd Schrader, "On the origin and removal of interference patterns in coated multimode fibres," Proc. SPIE 9912, Advances in Optical and Mechanical Technologies for Telescopes and Instrumentation II, 991255 (22 July 2016); doi: $10.1117 / 12.2233676$

Event: SPIE Astronomical Telescopes + Instrumentation, 2016, Edinburgh, United Kingdom 


\title{
On the origin and removal of interference patterns in coated multimode fibres
}

\author{
Yazmin Padilla Michel ${ }^{* a, b}$, Silvio Pulwer ${ }^{\mathrm{a}, \mathrm{b}}$, Pouneh Saffari ${ }^{\mathrm{c}}$, Viachaslau Ksianzou ${ }^{\mathrm{a}}$, Sigurd \\ Schrader $^{\mathrm{a}}$ \\ ${ }^{\text {a }}$ Faculty of Engineering and Natural Science, Technische Hochschule Wildau, Hochschulring 1, \\ Wildau, Germany 15745; ${ }^{b}$ Dept. of Industrial Engineering, University of Rome "Tor Vergata", Via \\ del Politecnico 1, Rome, Italy 00133; ${ }^{c}$ European XFEL GmbH, Notkestrasse 85, Hamburg, Germany \\ 22607.
}

\begin{abstract}
In this study, we present the experimental investigations on interference patterns, such as those already reported in VIMOS-IFU, and up to now no appropriate explanation has been presented. These interference patterns are produced in multimode fibres coated with acrylate or polyimide, which is the preferred coating material for the fibres used in IFUs. Our experiments show that, under specific conditions, cladding modes interact with the coating and produce interference. Our results show that the conditions at which the fibre is held during data acquisition has an impact in the output spectrum. Altering the positioning conditions of the fibre leads to the changes into the interference pattern, therefore, fibres should be carefully manipulated in order to minimise this potential problem and improve the performance of these instruments. Finally we present a simple way of predicting and modelling this interference produced from the visible to the near infrared spectra. This model can be included in the data reduction pipeline in order to remove the interference patterns.
\end{abstract}

These results should be of interest for the optimisation of the data reduction pipelines of instruments using optical fibres. Considering these results will benefit innovations and developments of high performance fibre systems.

Keywords: Instrumentation, multimode fibres, bending losses, fringing, interference, coating materials, data reduction

\section{INTRODUCTION}

Integral Field Units (IFUs) are nowadays widely used in large telescopes for three dimensional spectroscopy applications, i.e. acquisition of imaging and spectral information simultaneously. Despite of their wide advantage range, IFUs generally suffer from their complex design and bulky geometry. Although, extensive characterisation tests of these devices has been performed and publish (e.g. focal ratio degradation ${ }^{1}$, scattering and end-face roughness ${ }^{2}$; cross-talk and throughput $^{3}$; just to mention a very few of them), still there are subtle effects that need to be investigated in order to optimise their performance, obtain a better instrument footprint and its subsequent inclusion in the data reduction pipelines.

Such is the case of the fringing pattern reported in VIMOS-IFU ${ }^{4}$, where variable interference patterns affect the visible spectra, starting from $415 \mathrm{~nm}$ and increasing towards red wavelengths ${ }^{5,6}$. Up to now no appropriate explanation for this shortcoming has been presented, hindering an optimisation of its pipeline ${ }^{7}$.

Eliminating sources of interference in this instrument, it is well known that interference is produced in thinned CCDs, where the chip becomes transparent towards red wavelengths ( $750 \mathrm{~nm}$ onwards). For this reason the CCD of VIMOS was replaced for a deep depletion CCD to diminish the fringing ${ }^{8}$. Nevertheless, in Lagerholm et al. $2012{ }^{6}$ it is shown that the fringing persists starting from the violet range, and even with the new type of CCD. Therefore, they attribute this fringing to "flexure of the prism in the pseudo-slit" and/or "an etaloning effect at the fibre output prism".

*padilla-michel@th-wildau.de; www.th-wildau.de/photonik 
On the other hand, in the telecommunications and sensing field, there is an extensive list of publications presenting a clear dependency between bending losses and wavelength, either using single mode fibres ${ }^{9-11}$ or $\mathrm{MMF}^{12,13}$. This effect is known in the literature as whispering-gallery modes (for bent fibres) ${ }^{9}$ or oscillatory losses (for straight fibres) ${ }^{11}$. For the rest of this paper we will simply use the term interference pattern to refer the effect described before.

In this paper we present experimental results of how bending losses, using multimode fibres (MMF) coated with polyimide or acrylate, produce the interference pattern mentioned before. We also present a brief explanation of this effect and a method to predict the maxima or minima of the interference pattern. These results could be useful for the optimisation of the data reduction, and should be considered for existing and future instruments using optical fibres with the characteristic mentioned before.

\section{BACKGROUND (THEORY)}

The preferred fibre design (see figure 1) used to manufacture IFUs consist of a fused silica core, fluorinated silica cladding, and one or two polymeric coatings, which refractive index $\left(n_{3}\right)$ should be higher than the cladding refractive index $\left(n_{2}\right)$. This type of coating has two functions: 1) it is used to protect the glass of the fibre from the harsh environment; 2 ) it strips the cladding modes, due to its high refractive index.

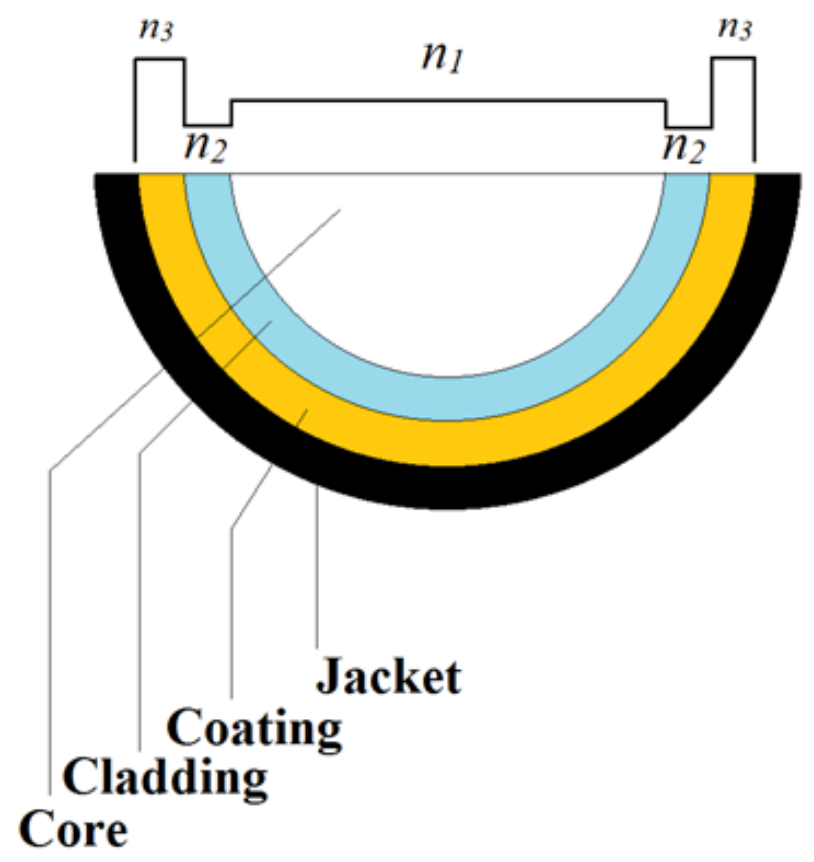

Figure 1. Schematic of cross section of a MMF with the design described previously. On top, the step refractive index profile of the MMF, where $n_{l}$ is the refractive index of the core, $n_{2}$ is the refractive index of the cladding and $n_{3}$ the refractive index of the coating. The jacket can be made ether of Nylon or Tefzel ${ }^{\mathrm{TM}}$, but it is not always used.

MMFs can carry thousands of modes depending on the core diameter and wavelength, but only a percentage of these core modes will propagate as cladding modes (e.g., $2.4 \%$ at $\lambda=1550 \mathrm{~nm})^{14}$. In figure 2 it is shown a numerical simulation, made with COMSOL Multiphysics, of the electromagnetic field propagating along a MMF with the design mentioned before, i.e. a silica fibre coated with polyimide. In the simulation it is shown that each mode propagates along the fibre core, but a part of it also propagates along the coating. There are no modes propagating along the cladding, because the coating has absorbed all of them.

According to the literature, when an optical fibre is bent, the evanescent field is deflected and hence the bending losses are produced ${ }^{9}$. Afterwards, the already deflected evanescent field will form a whispering-gallery mode (WGM) which is refracted from the cladding into the coating and reflected at the coating-air boundary ${ }^{10}$. The WGM keeps bouncing along the fibre bend. 


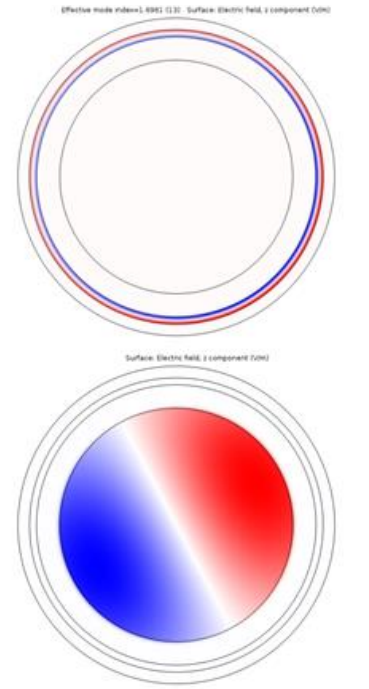

LP11
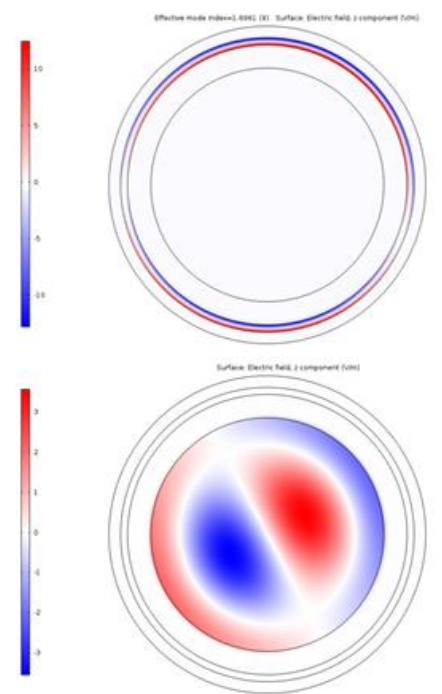

$\mathbf{L P}_{12}$
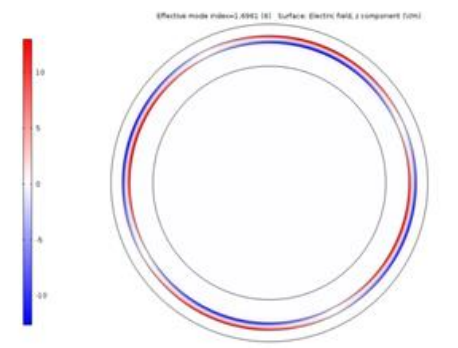

Coating modes
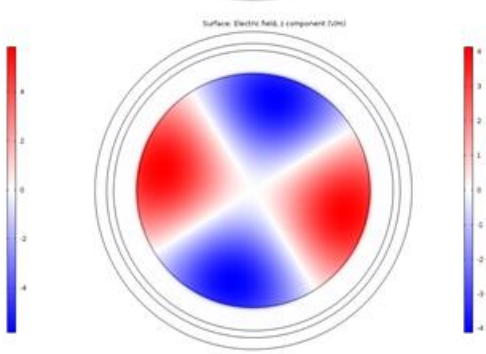

Core modes

$\mathbf{L P}_{21}$

Figure 2. Numerical simulations made with COMSOL Multiphysics. The core/cladding/coating outer diameters of the simulated MMF are 200/240/270 $\mu \mathrm{m}$ and the refractive indexes are 1.458/1.442/1.696 respectively. On the bottom, it is shown the propagation of the $\mathrm{LP}_{11}, \mathrm{LP}_{12}$ and $\mathrm{LP}_{21}$ modes along the fibre core. On the top, the simulation of the same modes but propagating along the coating. The figure shows only three of the 3200 modes (at $\lambda=1500 \mathrm{~nm}$ ) that this fibre can accept.

From this perspective, the simulations of the electromagnetic field of a big-core MMF in such a detail, consumes a big amount of resources (software time). Therefore, this issue will be addressed from the geometrical optics approach, which is valid for explaining bending losses in optical slabs ${ }^{15}$.

In Padilla et al. $2012^{22}$, it is shown that fibres which $\mathrm{n}_{3}$ is lower than $\mathrm{n}_{2}$, have very low attenuation (up to $0.037 \mathrm{~dB}$ at $0.48 \mathrm{GPa}$ ), because at the cladding-coating boundary the cladding modes are reflected, therefore most of the power remains between the core and cladding. On the other hand, the attenuation curve of fibres, which $n_{3}$ is higher than $n_{2}$, is exponential-like (up to $1.16 \mathrm{~dB}$ at $0.48 \mathrm{GPa}$ ). From the geometrical optics perspective, in this case, the cladding modes are refracted into the coating, reflected at the coating-air boundary, and partially reflected again at the cladding-coating boundary. The path that these modes describe to propagate along the cladding and coating resembles a Fabry-PerotEtalon (see figure 3).

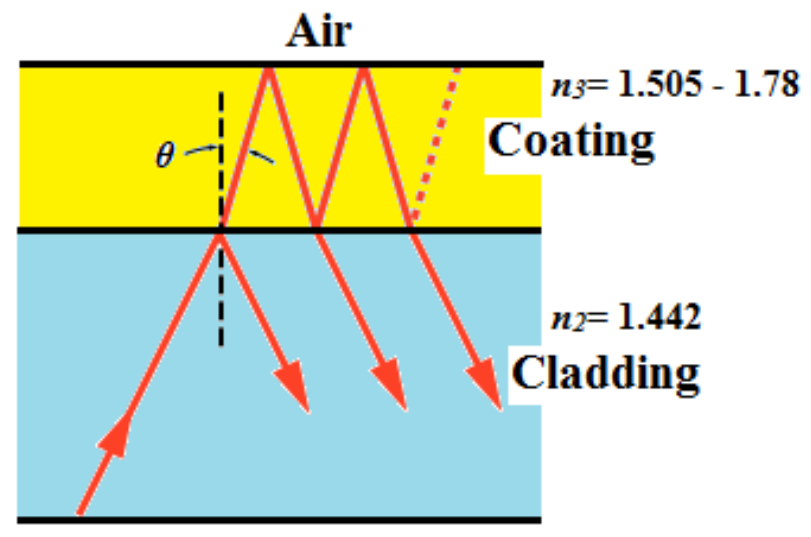

Figure 3. Schematic of the two outer layers of a MMF resembling an etalon. The red arrows show the path followed by cladding modes refracted in to the coating and bouncing between coating-air and coating-cladding boundaries.

In section 2.1 it will be explained how, in the case of a MMF, the higher-order modes traveling along the core loose guidance, due to fibre bending, and interfere with themselves producing the fringing patterns mentioned before. 


\subsection{Geometrical optics approach}

When a MMF is bent, the meridional modes, which incidence angle is near the critical angle $\theta_{c}$, are the most affected. These modes are refracted from the fibre core into the cladding in form of divergent beams, which divergence angle $\theta_{d i v}$ is calculated as ${ }^{16,17}$ :

$$
\theta_{d i v}=\cos ^{-1}\left(\frac{R-a}{R+a}\right)
$$

Where $R$ is the bending radius, and $a$ is the core radius (see figure 4). Once $\theta_{d i v}$ is calculated, it is easy to know the rest of the angles at which the divergent beam strikes the cladding-coating boundary $\left(\theta_{1}\right)$ and it is refracted in to the coating $\left(\theta_{2}\right)$ as follows:

$$
\begin{gathered}
\theta_{1}=90^{\circ}-\theta_{d i v} \\
\theta_{2}=\sin ^{-1}\left(\frac{n_{2} \sin \theta_{1}}{n_{3}}\right)
\end{gathered}
$$

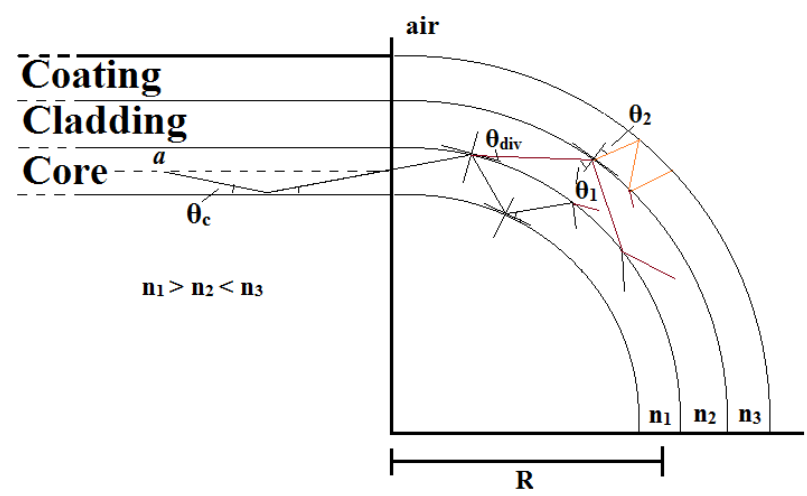

Figure 4. Schematic of a bent fibre and the propagation of only one divergent beam. Core, cladding and coating refractive indexes are $n_{1}, n_{2}$ and $n_{3}$ respectively. $\theta_{d i v}$ is the divergence angle defined in equation $1, \theta_{c}$ is the critical angle, and $a$ is the core radius.

Once $\theta_{d i v}, \theta_{1}$, and $\theta_{2}$ are known, it is straightforward to calculate the optical path difference of the refracted modes, and hence, the free spectral range $(F S R)$ of the etalon $(\Delta \tilde{\mathrm{v}})$ in $\left[\mathrm{cm}^{-1}\right]$ as follows: ${ }^{11}$

$$
\Delta \tilde{\mathrm{v}}=\left\{\left(2 C_{t h} / \cos \theta_{2}\right)\left[n_{3}(\lambda)-n_{2}(\lambda) \sin \theta_{1} \sin \theta_{2}\right]\right\}^{-1}
$$

where $C_{t h}$ is the coating thickness; $n_{2}(\lambda)$ and $n_{3}(\lambda)$ are the wavelength-dependent refractive indices of cladding and coating respectively.

\section{METHOD OF MEASUREMENT}

For our experiment, a Fourier transform spectrometer (MATRIX-F FT-IR from Bruker Optics) was used. The feeding spot of the spectrometer has a diameter of $\varnothing=3 \mathrm{~mm}$, in order to illuminate the whole fibre connector, and a fixed numerical aperture (NA) of 0.22 , which matches the NA of the fibres used for this experiment. This setup was chosen, because at the focal plane of a telescope, the entire input face of an IFU, including the core, cladding and coating of each fibre, is illuminated. Therefore, we want to reproduce the same feeding conditions.

All the fibre samples (see table 1) were $3 \mathrm{~m}$ long and a mirror cut was made on each fibre end using a diamond scribe tool. Once the fibre was cut and cleaned with isopropanol, it was preassembled into a SMA connector and hold with a 
pressure adapter (see figure 5), ensuring that the output hole of the connector were the closest to the fibre diameter. This also prevents that the fibre is tilted when it is connected to the spectrometer input/output ports.

Table 1. Fibre parameters. Every fibre has a core of fused silica and a cladding of fluorine-doped fused silica. The refractive indices information was provided by the manufacturers. All the fibres have a NA $=0.221 \pm 0.001$.

\begin{tabular}{|c|c|c|c|c|}
\hline \multirow[b]{2}{*}{ Coating material } & \multicolumn{3}{|c|}{ Refractive index at $600 \mathrm{~nm}$} & \multirow[b]{2}{*}{$\begin{array}{l}\text { Core/cladding/coating } \\
\text { outer diameters }[\mu \mathrm{m}]\end{array}$} \\
\hline & $\begin{array}{c}\text { Core } \\
n_{1}\end{array}$ & $\begin{array}{c}\text { Cladding } \\
n_{2}\end{array}$ & $\begin{array}{c}\text { Coating } \\
n_{3}\end{array}$ & \\
\hline Single coating acrylate 1 (IRA14) & 1.458 & 1.442 & 1.540 & $200 / 240 / 375$ \\
\hline Secondary coating acrylate (IRA136) & 1.458 & 1.442 & $\begin{array}{l}1.505 \text { (I) } \\
1.541 \text { (II) }\end{array}$ & $200 / 240 / ? / 375$ \\
\hline Polyimide 1 (IRPI) & 1.458 & 1.442 & 1.7 & $\begin{array}{l}200 / 220 / 245 \\
200 / 240 / 252 \\
200 / 240 / 265\end{array}$ \\
\hline Polyimide 2 (FBP) & 1.458 & 1.442 & 1.78 & $300 / 330 / 370$ \\
\hline Polyimide 3 (FTUV) & 1.458 & 1.442 & 1.7 & $266 / 292 / 318$ \\
\hline
\end{tabular}

After the fibre was connected to the spectrometer, a reference spectrum was obtained with the fibre relaxed. Subsequently, the fibre was coiled around the bending tool forming a loop and then, fastened using magic tape. The fibre is not disconnected during the measurements to ensure the same feeding conditions. The bending tool has four cylinders of different diameters. Changing the bending radius will lead to variations of the $\theta_{d i v}$, as shown in equation 1. Finally, the spectrum obtained from the bent fibre was normalised, i.e. divided by the reference spectrum, and finally, an interference pattern is visible in the resulting normalised spectrum (transmittance).
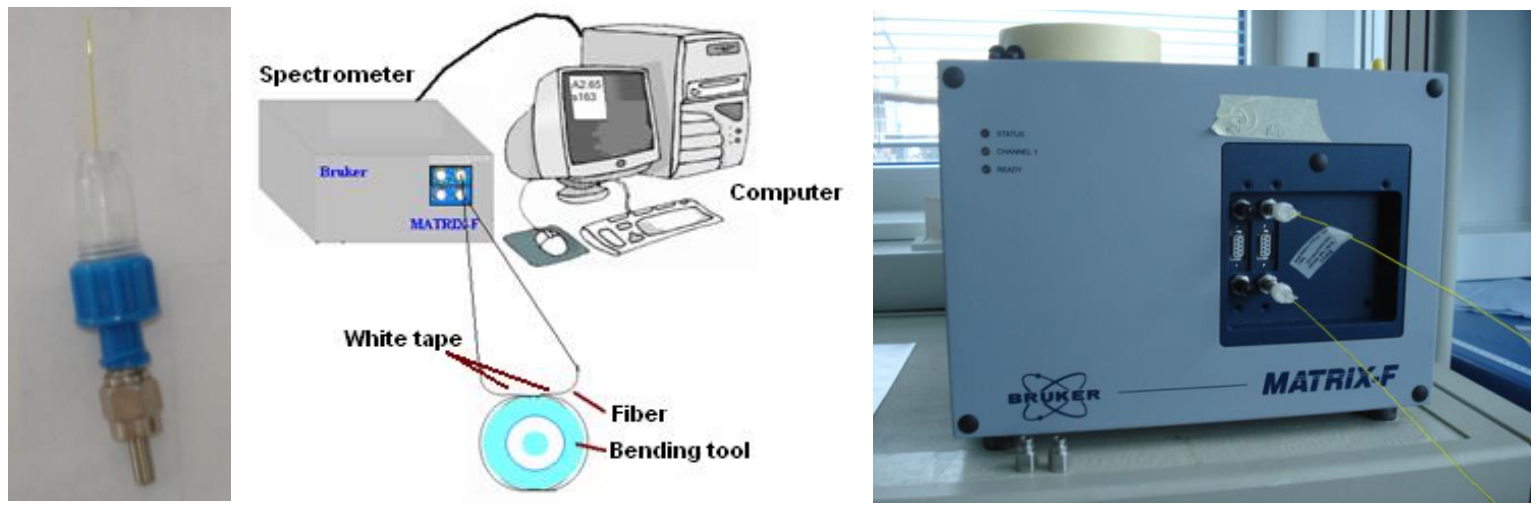

Figure 5. On the left, a pressure adapter with a SMA connector and a polyimide fibre assembled. On the centre, schematic of a fibre coiled around a bending tool and connected to the spectrometer. On the right, a close-up view to a fibre connected to the input/output ports of the spectrometer.

\section{RESULTS AND DISCUSSION}

The results of our experiment are sorted by fibre characteristics, i.e. outer diameter, hydrogen content, coating material and manufacturer. This is to ensure that interference is produced in every fibre which $n_{3}$ is higher than $n_{2}$, independently of any other parameters.

As mentioned before, the transmittance is calculated with equation 5 as: 


$$
T=\frac{P_{b f}}{P_{r f}}
$$

where $T$ is the fibre transmittance, $P_{r f}$ is the power transmitted by the relaxed fibre, and $P_{b f}$ is the power transmitted by the bent fibre.

As mentioned before, every fibre was bent at two different bending radii. As expected, the smaller the bending radius, the lower the transmittance, because a bigger percentage of core modes are deflected. The average FSR and the wavelength shift between the maxima of both curves are calculated considering the first five maxima from longer to shorter wavelengths.

\subsection{Fibres coated with polyimide}

In this section, two fibres with the same characteristics, i.e. material, core/cladding outer diameters, and NA, are compared. The only one difference between these two fibres is the coating thickness, $6 \mu \mathrm{m}$ (figure 6 left) and $12.5 \mu \mathrm{m}$ (figure 6 right).
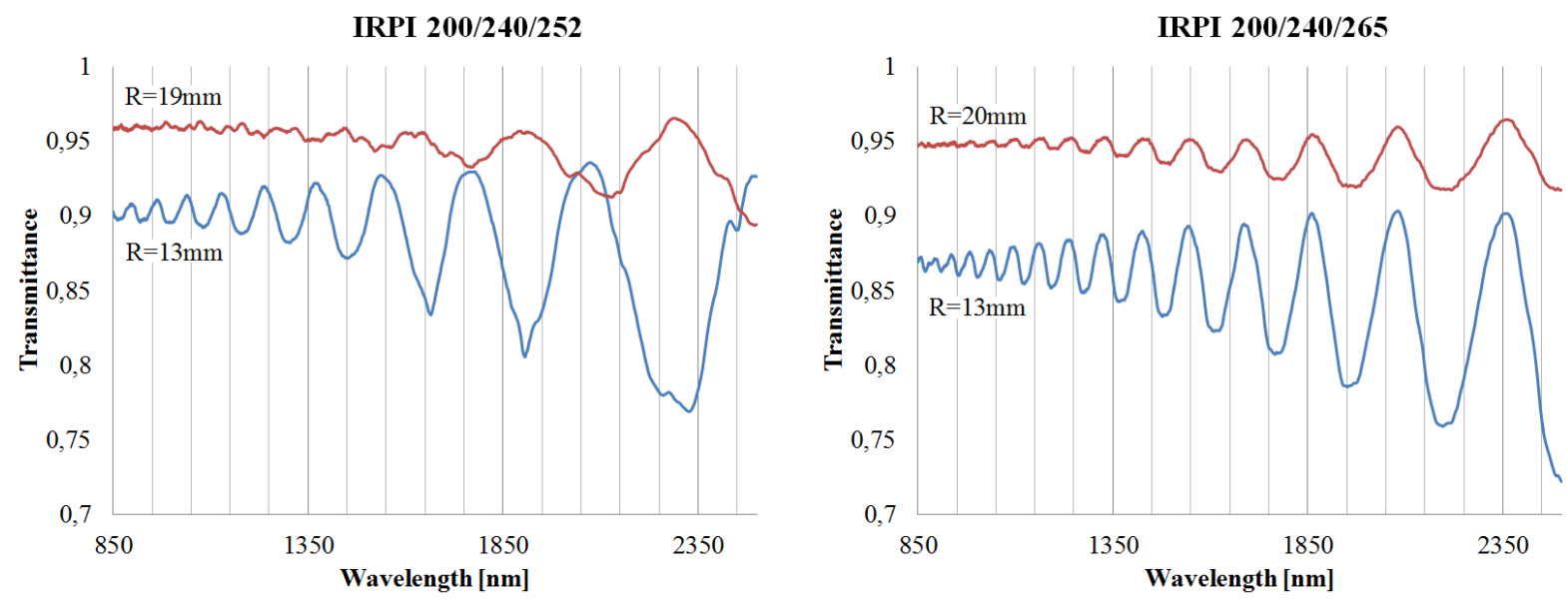

Figure 6. Transmittances of two bent MMFs, at different bending radius.

In table 2 it is shown that the differences between resulting $\theta_{d i v}$, when changing the bending radius, are really small $\left(<2^{\circ}\right)$, therefore, the wavelength shift between spectra of the same fibre, but at different bending radius, should be small as well. This is the case of the fibre which $C_{t h}=12.5 \mu \mathrm{m}$, where the wavelength shift is just $1.6 \mathrm{~nm}$, and the FSR does not change with the bending radius, proving that it depends only on $C_{t h}$.

Table 2. Characteristics of the fibre spectra shown in figure 6 .

\begin{tabular}{|c|c|c|c|c|c|c|}
\hline \multirow[b]{2}{*}{$\begin{array}{c}\text { Fibre } \\
\text { (coating outer } \\
\text { diameter) }\end{array}$} & \multirow[b]{2}{*}{$C_{t h}[\mu \mathrm{m}]$} & \multicolumn{2}{|c|}{$\boldsymbol{\theta}_{\text {div }}\left[{ }^{\circ}\right]$} & \multicolumn{2}{|c|}{ Average $F S R[\mathrm{~nm}]$} & \multirow[b]{2}{*}{$\begin{array}{c}\text { Wavelength Shift } \\
{[\mathrm{nm}]}\end{array}$} \\
\hline & & $\begin{array}{l}\text { Small R } \\
(13 \mathrm{~mm}) \\
(13 \mathrm{~mm})\end{array}$ & $\begin{array}{c}\text { Big R } \\
(19 \mathrm{~mm}) \\
(20 \mathrm{~mm})\end{array}$ & $\begin{array}{l}\text { Small R } \\
(13 \mathrm{~mm}) \\
(13 \mathrm{~mm})\end{array}$ & $\begin{array}{c}\text { Big R } \\
(19 \mathrm{~mm}) \\
(20 \mathrm{~mm})\end{array}$ & \\
\hline IRPI (252) & 6 & 9.98 & 8.27 & 287.75 & 242.5 & 124.2 \\
\hline IRPI (265) & 12.5 & 9.97 & 8.06 & 203.75 & 203.5 & 1.6 \\
\hline
\end{tabular}

On the other hand, the fibre which $C_{t h}=6 \mu \mathrm{m}$, the wavelength shift is considerably big, the average $F S R$ changes, and the oscillations are not well defined towards shorter wavelengths. This could be due to an incorrect gripping on the bending tool when measuring the transmittance at $R=19 \mathrm{~mm}$. Even so, both spectra suffer of interference and the FSR is inversely proportional to $C_{t h}$, in agreement to equation 4 . 


\subsection{Fibres coated with polyimide and different hydrogen content}

In this section, a dry fibre (optimised for VIS/NIR transmission) and a solarisation resistant fibre (optimised for UV/VIS transmission), are compared. Both fibres are made of the same material and have equal NA. The differences are the outer diameters and hydrogen content. As expected, the UV-optimised fibre show strong noise around $1400 \mathrm{~nm}$ and after $2100 \mathrm{~nm}$ due to its high content of hydrogen.

IRPI 200/220/245

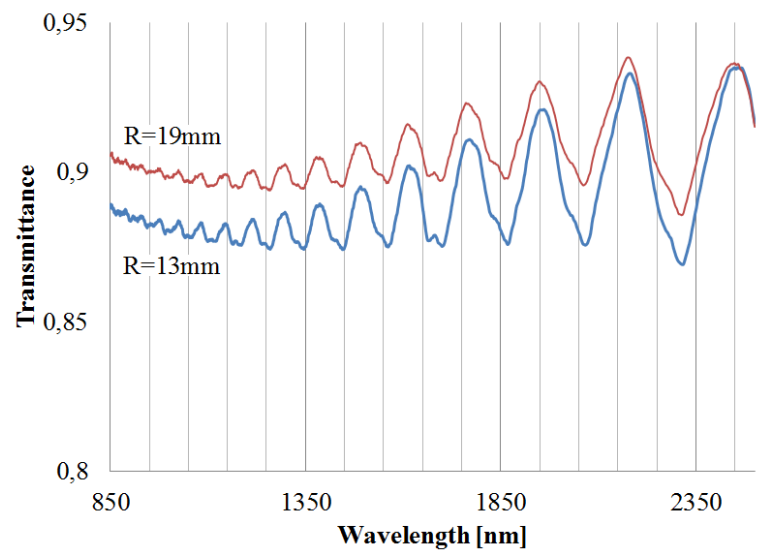

FTUV 266/292/318

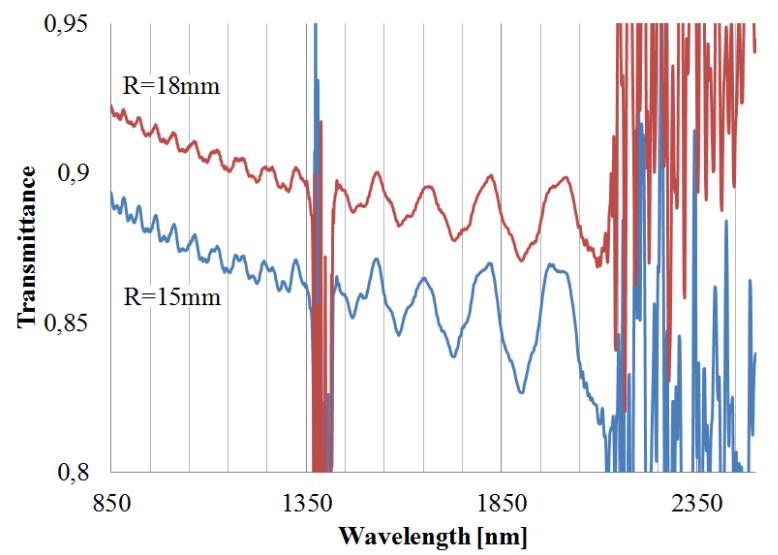

Figure 7. Transmittances of two bent MMFs, at different bending radii. On the left, spectra of a dry MMF. On the right, spectra of a hydrogen-loaded fibre. This fibre is identical of those used to manufacture VIRUS-P IFU ${ }^{19}$.

In table 3 it is shown that the resulting $\theta_{d i v}$ from the different bending radii, is again very small $\left(<2^{\circ}\right)$, and in both cases a small wavelength-shift is produced, i.e. $8.2 \mathrm{~nm}$ for the thinner fibre and $12.4 \mathrm{~nm}$ for the thicker one. Since, according to the specifications of the manufacturer, the $C_{t h}$ of the fibres is only $0.5 \mu \mathrm{m}$, the FSR should be similar. However the FSR difference is $>60 \mathrm{~nm}$. Therefore, we have measured again the outer coating diameter of the fibre with a microscope, and the result was $243 \mu \mathrm{m}$, i.e. the $C_{t h}=11.5 \mu \mathrm{m}$, explaining why the $F S R$ of this fibre is wider than expected.

Table 3. Characteristics of the spectra shown in figure 7.

\begin{tabular}{|c|c|c|c|c|c|c|}
\hline \multirow[b]{2}{*}{$\begin{array}{c}\text { Fibre } \\
\text { (coating outer } \\
\text { diameter) }\end{array}$} & \multirow[b]{2}{*}{$C_{t h}[\mu \mathrm{m}]$} & \multicolumn{2}{|c|}{$\boldsymbol{\theta}_{\text {div }}\left[{ }^{\circ}\right]$} & \multicolumn{2}{|c|}{ Average $F S R[\mathrm{~nm}]$} & \multirow[b]{2}{*}{$\begin{array}{c}\text { Wavelength Shift } \\
{[\mathrm{nm}]}\end{array}$} \\
\hline & & $\begin{array}{l}\text { Small } R \\
(13 \mathrm{~mm}) \\
(15 \mathrm{~mm})\end{array}$ & $\begin{array}{c}\text { Big } R \\
(19 \mathrm{~mm}) \\
(18 \mathrm{~mm})\end{array}$ & $\begin{array}{l}\text { Small } R \\
(13 \mathrm{~mm}) \\
(15 \mathrm{~mm})\end{array}$ & $\begin{array}{c}\text { Big } R \\
(19 \mathrm{~mm}) \\
(18 \mathrm{~mm})\end{array}$ & \\
\hline IRPI (245) & 12.5 & 10.06 & 8.27 & 210.25 & 209.25 & 8.2 \\
\hline FTUV (318) & 13 & 10.7 & 9.78 & 136.25 & 147 & 12.4 \\
\hline
\end{tabular}

\subsection{Fibres coated with acrylate}

In this section, two fibres with equal outer diameters and made with the same materials are compared. The difference between them is that the fibre labelled IRA14 has only one acrylate coating and the fibre labelled IRA136 has a double coating. The manufacturer has provided the $n_{3}$ of each layer (see table 1), but not the outer diameter of the inner coating. The inner coating is more elastic (Young Modulus of $\sim 7 \mathrm{MPa}$ ) than the outer coating (Young Modulus of $\sim 2100 \mathrm{MPa}$ ) ${ }^{18}$. When this type of fibre is bent, micro-bendings are produced between both coatings, increasing the bending losses (see figure 8 right). 

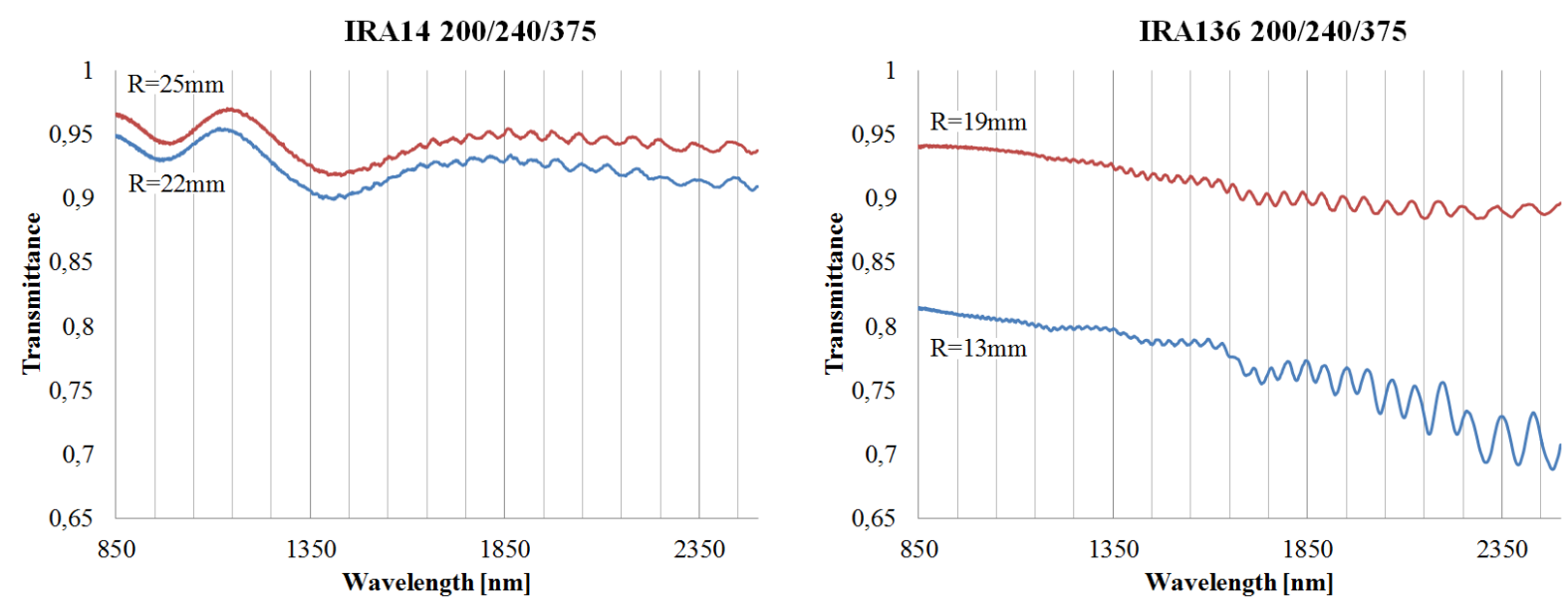

Figure 8. Spectra of two bent MMFs coated with acrylate. On the left, the spectra of a bent fibre with one coating. On the right, spectra of a bent fibre with a double coating.

In table 4 it is shown that even if the fibres have the same coating thickness, the double-coated fibre has a smaller FSR than the single-coated one. Considering the FSR difference between fibres and knowing the $n_{3}$ of the inner and outer coating, it would be possible to calculate the thickness of the inner coating of the fibre IRA136 by the geometrical optics approach.

Table 4. Characteristics of the spectra shown in figure 8.

\begin{tabular}{|c|c|c|c|c|c|c|}
\hline \multirow[b]{2}{*}{$\begin{array}{c}\text { Fibre } \\
\text { (coating outer } \\
\text { diameter) }\end{array}$} & \multirow[b]{2}{*}{$C_{t h}[\mu \mathrm{m}]$} & \multicolumn{2}{|c|}{$\boldsymbol{\theta}_{\text {div }}\left[{ }^{\circ}\right]$} & \multicolumn{2}{|c|}{$F S R[\mathrm{~nm}]$} & \multirow[b]{2}{*}{$\begin{array}{c}\text { Wavelength Shift } \\
{[\mathrm{nm}]}\end{array}$} \\
\hline & & $\begin{array}{l}\text { Small } R \\
(22 \mathrm{~mm}) \\
(13 \mathrm{~mm})\end{array}$ & $\begin{array}{c}\text { Big } R \\
(25 \mathrm{~mm}) \\
(19 \mathrm{~mm})\end{array}$ & $\begin{array}{l}\text { Small } R \\
(22 \mathrm{~mm}) \\
(13 \mathrm{~mm})\end{array}$ & $\begin{array}{c}\text { Big } R \\
(25 \mathrm{~mm}) \\
(19 \mathrm{~mm})\end{array}$ & \\
\hline IRA14 (375) & 67.5 & 7.68 & 7.21 & 81 & 80.75 & 5.2 \\
\hline IRA136 (375) & $67.5(/ 2)$ & 9.95 & 8.26 & 76.25 & 75.25 & 10 \\
\hline
\end{tabular}

\subsection{Fibres coated with another type of polyimide}

The results presented in sections 4.1 to 4.3 have been made with MMF provided by FiberTech GmbH. In order to prove that this is not a quality issue of the manufacturer, in this section we present the spectra of a MMF coated with polyimide provided by Polymicro Technologies ${ }^{\mathrm{TM}}$. This fibre has a $n_{3}$ higher than that of the fibres from FiberTech GmbH (see table 1), besides the colour of its coating is brown burnt, unlike the bright-golden coating of FiberTech GmbH fibres. This means that this fibre has been cured for a longer time to a higher temperature, changing the optical and mechanical properties of the polyimide. Even so, the transmittance of this fibre also suffers of interference patterns like those measured before (see figure 9).

Table 5. Characteristics of the spectra shown in figure 9.

\begin{tabular}{|c|c|c|c|c|c|c|}
\hline \multirow[b]{2}{*}{$\begin{array}{c}\text { Fibre } \\
\text { (coating outer } \\
\text { diameter) }\end{array}$} & \multirow[b]{2}{*}{$C_{t h}[\mu \mathrm{m}]$} & \multicolumn{2}{|c|}{$\boldsymbol{\theta}_{d i v}\left[^{\circ}\right]$} & \multicolumn{2}{|c|}{$F S R[\mathrm{~nm}]$} & \multirow[b]{2}{*}{$\begin{array}{c}\text { Wavelength Shift } \\
{[\mathrm{nm}]}\end{array}$} \\
\hline & & $\begin{array}{l}\text { Small } R \\
(16 \mathrm{~mm})\end{array}$ & $\begin{array}{c}\text { Big } R \\
(19 \mathrm{~mm})\end{array}$ & $\begin{array}{l}\text { Small } R \\
(16 \mathrm{~mm})\end{array}$ & $\begin{array}{c}\operatorname{Big} R \\
(19 \mathrm{~mm})\end{array}$ & \\
\hline FBP (370) & 20 & 11 & 10.11 & 81 & 79.5 & 2 \\
\hline
\end{tabular}


FBP 300/330/370

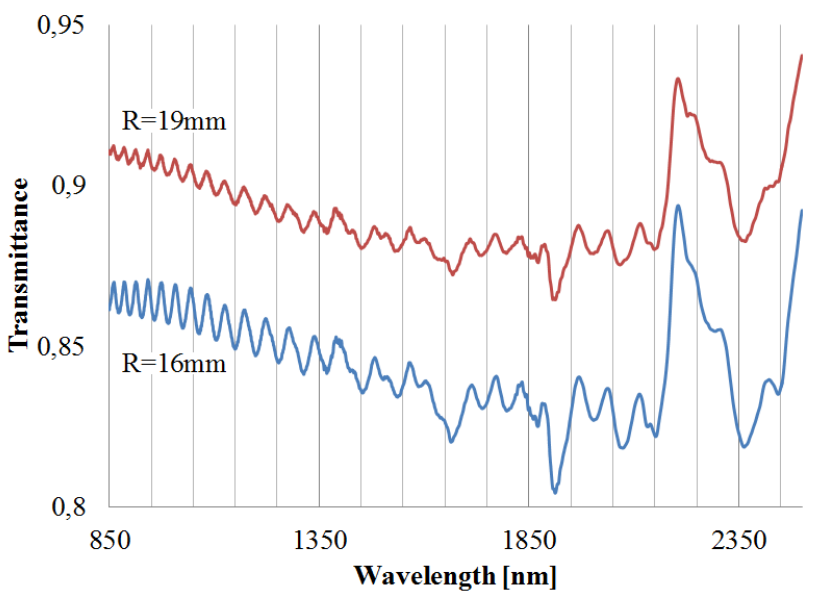

Figure 9. Spectra of a MMF coated with a different type of polyimide.

\section{DISCUSSION}

Back to the fringing pattern reported in VIMOS-IFU, Jullo et al. $2008^{5}$ presented a set of normalised sky spectra taken at different rotation angles of the instrument. These spectra clearly show a wavelength shift $<10 \mathrm{~nm}$, between the calibration spectrum taken at $26.6^{\circ}$ and the spectra taken at $83^{\circ}$ and at $116^{\circ}$. This is a very similar wavelength shift shown before in our results; proving that when the instrument rotates, the fibres of the IFU are bent forming a $R$ of $\sim 20 \mathrm{~mm}$. VIMOS is a very complicated instrument and the fibre bundles are placed in a very intricate way, even so, a closer look to the fibre bundles during the rotation of the instrument, will be determinant to find the place where the fibres are bent and to measure the bending radius at different rotation angles.

IRA136 200/240/375

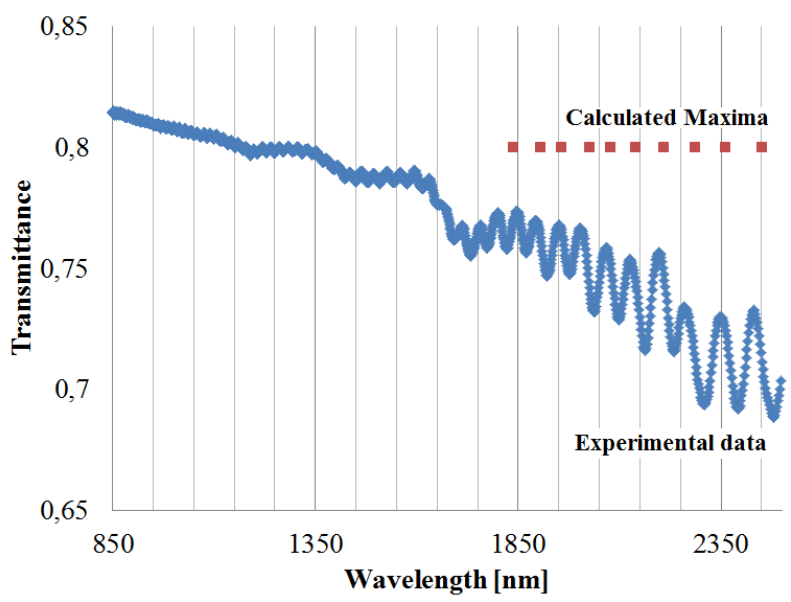

Figure 10. Spectra of a MMF with double acrylate coating (blue diamonds) and the maxima calculated using equation 4 (red squares).

On the other hand, Lagerholm et al. $2012^{6}$ reported that the fringing pattern resembles an etalon with a thickness varying from 3 to $10 \mu \mathrm{m}$ and possibly composed of multiple layers, but the source of this etaloning could not be found. Taking a closer look to the design of VIMOS fibres, in Prieto et al $2000^{23}$ is reported that the chosen fibres have a core of $100 \mu \mathrm{m}$, a doped silica cladding of $140 \mu \mathrm{m}$, an outer silica cladding of $170 \mu \mathrm{m}$, an inner soft epoxy layer, which outer diameter is not specified, and finally an outer strong epoxy layer of $250 \mu \mathrm{m}$. Unfortunately the refractive index of each layer are not mentioned in their paper, neither the epoxy material. Even so, with this information, we can assume a similar design to 
that of our fibre labelled IRA136, which has a soft inner coating and a harder outer one. Therefore, we used equation 4 to calculate the maxima of transmittance of the fibre labelled IRA 136 (see figure 10).

In figure 10 it is shown a good agreement between the experimental and calculated maxima, this confirms that it is possible to make a model of the interference patter using geometrical optics as long as $R$ can be measured accurately and, consequently, $\theta_{d i v}$ can be calculated. If all the angles mentioned in equations 1,2 , and 3 , can be calculated with precision, not only equation 4, but also Fresnel's equations can be used to make a model of the interference patterns produced by the fibres, and subsequently, included in the data reduction pipeline of the affected instruments.

\section{CONCLUSION}

We have shown that interference patterns in the transmittance are produced when MMFs are bent. These interference patterns are strongly dependent on refractive index, thickness and coating material; and they can be interpreted in the framework of geometrical optics like an etalon of multiple layers. Even if this issue can be consider an old issue of VIMOS-IFU, the type of fibres used for our experiment are also being used in other instruments such as VIRUS ${ }^{19}$, EDiFiSe $^{20}$, and will be used in other instrument for coming such as 4MOST ${ }^{21}$, just for mention a few of them. Therefore, these results should be taken into account for a better performance of the fibre-based IFUs.

\section{ACKNOWLEDGMENT}

To AiF Projekt GmbH (project number KF2014158NT3) for the founding, to Dr Mohamad Zoheidi from OBERON GmbH Fiber Technologies and to Alexander Heinze from LEONI Fiber Optics GmbH for providing the fibre samples and for allowing us to use their FT-IR Spectrometer and their lab facilities.

\section{REFERENCES}

[1] Ramsey, L. W. "Focal ratio degradation in optical fibers of astronomical interest." In Fiber optics in astronomy 3, 26-39 (1988).

[2] Haynes, D. M., Withford, M. J., Dawes, J. M., Lawrence, J. S., and Haynes, R., "Relative contributions of scattering, diffraction and modal diffusion to focal ratio degradation in optical fibres," Monthly Notices of the Royal Astronomical Society 414(1), 253-263 (2011).

[3] Bryant, J. J., O'Byrne, J. W., Bland-Hawthorn, J., and Leon-Saval, S. G., "Characterization of hexabundles: initial results," Monthly Notices of the Royal Astronomical Society 415(3), 2173-2181 (2011).

[4] LeFevre, O., Vettolani, G., Maccagni, D., Mancini, D., Picat, J. P., Mellier, Y., Mazure, A., Saisse, M., Cuby, J. G., Delabre, B., Garilli, B., Hill, L., Prieto, E., Arnold, L., Conconi, P., Cascone, E., Mattaini, E., and Voet, C., "VIRMOS: visible and infrared multiobject spectrographs for the VLT," Proc. SPIE 3355, 8 (1998).

[5] Jullo, E., Christensen, L., Smette, A., Bagnulo, S., Izzo, C., and Marconi, G. "The Origin of Fringing in the VIMOS IFU," In The 2007 ESO Instrument Calibration Workshop, Springer Berlin Heidelberg, 343-346 (2008).

[6] Lagerholm, C., Kuntschner, H., Cappellari, M., Krajnović, D., McDermid, R. M., and Rejkuba, M., "A way to deal with the fringe-like pattern in VIMOS-IFU data," Astronomy \& Astrophysics 541, A82 (2012).

[7] Mieske, S., Selman, F., "VIMOS User Maual,", ESO, 26 February 2015, <http://www.eso.org/sci/facilities/paranal/instruments/vimos/doc/VLT-MAN-ESO-14610-3509_v98.pdf> (06 June 2016).

[8] Hammersley, P., Christensen, L., Dekker, H., Izzo, C., Selman, F., Bristow, P., Bourget, P., Castillo, R., Downing, M., Haddad, N., Hilker, M., Lozon, J.-L., Lucuix, C., Mainieri, V., Mieske, S., Reinero, C., Rejkuba, M., Rojas, C., Sánchez-Janssen, R., Smette, A., Urrutia Del Rio, J., Valenzuela, J., and Wolff, B., "Upgrading VIMOS," The Messenger, 142, 8-11 (2010). 
[9] Harris, A. J., and Castle, P. F., "Bend loss measurements on high numerical aperture single-mode fibers as a function of wavelength and bend radius," Journal of Lightwave Technology 4(1), 34-40 (1986).

[10] Morgan, R., Barton, J. S., Harper, P. G., and Jones, J. D., "Wavelength dependence of bending loss in monomode optical fibers: effect of the fiber buffer coating," Optics letters 15(17), 947-949 (1990).

[11] Sharma, A., Powell-Friend, Y., George, T., and Phillips, L. "Oscillatory light loss within the cladding of a straight optical fiber: comparison with whispering-gallery modes". Optics letters 23(4), 262-264 (1998).

[12] Padilla Michel, Y., Steglich, P., Schrader, S., and Casalboni, M., "Whispering-Gallery Modes in Multimode Fibres: Experimental evidence and ray tracing model," In The European Conference on Lasers and ElectroOptics, Optical Society of America, CH_P_10 (2015).

[13]Moon, S., and Chen, Z., "Mode-filtered large-core fiber for optical coherence tomography," Applied optics, 51(34), 8262-8270 (2012).

[14] Gloge, D., "Weakly guiding fibers," Applied Optics 10(10), 2252-2258 (1971).

[15] Snyder, A. W., and Love, J. D., "Reflection at a Curved Dielectric Interface-Electromagnetic Tunneling," IEEE Transactions on Microwave Theory and Techniques, 23(1), 134-141 (1975).

[16] Badar, A. H., Maclean, T. S. M., Gazey, B. K., Miller, J. F., and Ghafoori-Shiraz, H., "Radiation from circular bends in multimode and single-mode optical fibres," In IEE Proceedings J Optoelectronics, 136 (3), 147-151 IET (1989).

[17]Badar, A. H., and Maclean, T. S. M., "Transition and pure bending losses in multimode and single-mode bent optical fibres,” In IEE Proceedings J Optoelectronics, 138 (4), 261-268 IET (1991).

[18] Padilla Michel, Y., Lucci, M., Casalboni, M., Steglich, P., and Schrader, S., "Mechanical Characterisation of the Four Most Used Coating Materials for Optical Fibres," Proceedings of the 3rd International Conference on Photonics, Optics and Laser Technology 1, 96-102 (2015).

[19]Hill, G. J., and MacQueen, P. J., "VIRUS: an ultracheap 1000-object IFU spectrograph,” Proc. SPIE 4836, 306312 (2002).

[20] García-Lorenzo, B., Fuensalida, J. J., Rodríguez-Hernández, M. A. C., Alonso, A., Barreto, M., Gracia-Temich, F., Martín, Y., Rodríguez, L. F., Viera, T., Padilla Michel, Y., Fernández, A., and Escobar-Romero, J. F. M., "EDiFiSE: equalized and diffraction-limited field spectrograph experiment," Proc. SPIE 7014, 70144B (2008).

[21] De Jong, R. S., Bellido-Tirado, O., Chiappini, C., Depagne, E., Haynes, R., Johl, D., Schnurr, O., Schwope, A., Walcher, J., Dionies, F., Haynes D. M., Kelz, A., Kitaura, F. S., Lamer, G., Minchev, I., Müller, V., Nuza, S. E., Olaya, J.-C., Piffl, T., Popow, E., Steinmetz, M., ; Ural, U., Williams, M., Winkler, R., Wisotzki, L., Ansorge, W. R., Banerji, M., Gonzalez Solares, E., Irwin, M., Kennicutt, R. C., King, D., McMahon, R. G., Koposov, S., Parry, I. R., Sun, D, Walton, N. A., Finger, G., Iwert, O., Krumpe, M., Lizon, J.-L., Vincenzo, M., Amans, J.-P., Bonifacio, P., Cohen, M., Francois, P., Jagourel, P., Mignot, s. B., Royer, F., Sartoretti, P., Bender, R., Grupp, F., Hess, ..., and Boland, W., "4MOST: 4-metre multi-object spectroscopic telescope," Proc. SPIE 8446, 84460T (2012).

[22] Padilla Michel, Y., Zoheidi, M., Roth, M. M., Haynes, R., and Olaya, J.-C., "Applied stress on coated multimode optical fibres: a different point of view to bending losses," Proc. SPIE 8450, 84503F (2012).

[23] Prieto, E., LeFevre, O., Saisse, M., Voet, C., and Bonneville, C., "Very wide integral field unit of VIRMOS for the VLT: design and performances," Proc. SPIE 4008, 510 (2000). 